\title{
Characterization and study of the effect of sulfate and bicarbonate on the catalytic activity of $\beta$-lactamase OXA-143 (P227S).
}

\section{Rafael Rospendowski, Víctor U. Antunes, Denize C. Favaro.}

\begin{abstract}
The aim of this study was to determine the hydrodynamic parameters of $\beta$-lactamase OXA-143(P227S) and to evaluate the effect of sulfate and bicarbonate salts on the penicillinase activity of the enzyme. Using chromatographic and spectroscopic techniques, it was possible to determine its diffusion coefficient, hydrodynamic radius and molecular mass, showing that mutant P227S has a tertiary structure of globular shape. The kinetic results showed an increase in catalytic activity only in the assay with increasing bicarbonate concentration. The bicarbonate supplementation also increases the thermal stability of the enzyme, which doesn't occur with the addition of sulfate.
\end{abstract}

\section{Key words:}

Antibiotic resistance, $\beta$-lactamase, enzyme kinetics.

\section{Introduction}

Class D $\beta$-lactamases known as oxacillinases (OXAs) are characterized by their similar folds comprising two domains. The first domain is formed by two $\alpha$-helices and a six-stranded $\beta$-sheet, while the second domain is exclusively $\alpha$-helical. The active site is located at the interface between these two domains. Carbapenemhydrolyzing Class D B-lactamases (CHDLs) confer to Gram-negative bacteria Acinetobacter baumannii resistance to carbapenems the so-called "last resort" antibiotics. These enzymes are recently-evolved variants of oxacillinases and are capable of conferring carbapenem resistance to this bacterium. ${ }^{1}$

In this study, we focus on the investigation of the influence of ionic strength and concentration of bicarbonate to the catalytic efficiency of the mutant OXA143(P227S) against ampicillin. We also performed an investigation of the enzyme hydrodynamic parameters.

\section{Results and Discussion}

The purified protein was used to obtain the hydrodynamic parameters by combining analytical gel filtration chromatography (AGF), size-exclusion chromatography coupled with multiangle light scattering detection (SECMALS), and dynamic light scattering (DLS), see Table 1. A Perrin factor near to 1 indicates that the enzyme is monomeric and has a globular or spherical tertiary structure under the studied conditions. ${ }^{1}$

Table 1. Hydrodynamic parameters of OXA-143(P227S).

\begin{tabular}{|c|c|c|c|c|}
\hline Techniques & $\operatorname{Rs}(\AA)$ & MM (kDa) & $D\left(x 10^{-7} \mathrm{~cm}^{2} \mathrm{~s}^{-1}\right)$ & $\begin{array}{l}\text { Perrin } \\
\text { factor }\end{array}$ \\
\hline AGF & $20.3 \pm 2$ a & $29.0^{b}$ & $1.1^{b}$ & \multirow{4}{*}{1.0} \\
\hline DLS & $16.8^{b}$ & $17.1^{\mathrm{b}}$ & $1.3 \pm 0.5^{a}$ & \\
\hline SEC-MALS & $20.3^{b}$ & $29.1 \pm 6^{a}$ & $1.1^{\mathrm{b}}$ & \\
\hline Predicted & 20.4 & $29.5^{c}$ & 1.1 & \\
\hline
\end{tabular}

a Data obtained experimentally at $25{ }^{\circ} \mathrm{C}$. ${ }^{\mathrm{b}}$ Data calculated by the Stokes-Einstein equation. ${ }^{\mathrm{c}}$ Data obtained ProtParam.

The enzymatic parameters ( $\mathrm{Km}$ and kcat) were obtained by UV-Vis spectroscopy (Table 2). It is observed an increase of the turnover-number ( $k_{\text {cat }}$ from 80 to $137 \mathrm{~s}^{-1}$ when the ionic strength was increased from 0 to $0.1 \mathrm{M}$ upon addition of sulfate. For the Michaelis constant $(\mathrm{Km})$, however, it is observed a random behaviour. In contrast, it is observed an increase in the catalytic efficiency $\left(\mathrm{k}_{\mathrm{cat}} / \mathrm{Km}\right)$ upon addition of bicarbonate. This enhancement in the activity was due to a combination of higher affinity (lower $\mathrm{Km}$ ) and higher turnover-number.

Table 2. OXA-143(P227S) kinetic parameters upon different concentrations of sulfate and bicarbonate.

\begin{tabular}{|c|c|c|c|c|c|c|}
\hline & \multicolumn{3}{|c|}{ Sulfate } & \multicolumn{3}{c|}{ Bicarbonate } \\
\hline $\begin{array}{c}\text { [Salt] } / \\
\mathbf{m M}\end{array}$ & $\begin{array}{c}\mathbf{k}_{\mathbf{c a t}} \\
\left(\mathbf{s}^{-1}\right)\end{array}$ & $\begin{array}{c}\mathbf{K m} \\
(\mu \mathrm{M})\end{array}$ & $\begin{array}{c}\mathbf{k}_{\mathbf{c a t}} / \mathbf{K m} \\
\left(\mu \mathrm{M}^{-1} \mathbf{s}^{-1}\right)\end{array}$ & $\begin{array}{c}\mathbf{k}_{\mathbf{c a t}} \\
\left(\mathbf{s}^{-1}\right)\end{array}$ & $\begin{array}{c}\mathbf{K m} \\
(\mu \mathrm{M})\end{array}$ & $\begin{array}{c}\mathbf{k}_{\mathbf{c a t}} / \mathbf{K m} \\
\left(\mu \mathrm{M}^{-1} \mathbf{s}^{-1}\right)\end{array}$ \\
\hline $\mathbf{0}$ & 80.1 & 49.3 & 1.6 & 80.1 & 49.3 & 1.6 \\
\hline $\mathbf{2 5}$ & 115.8 & 196.7 & 0.6 & 100.6 & 47.3 & 2.1 \\
\hline $\mathbf{5 0}$ & 124.9 & 79.9 & 1.6 & 110.4 & 44.5 & 2.5 \\
\hline $\mathbf{1 0 0}$ & 136.5 & 152,0 & 0.9 & - & - & - \\
\hline $\mathbf{2 0 0}$ & 110.2 & 73.65 & 1.5 & - & - & - \\
\hline
\end{tabular}

Inspection of Figures $1 \mathrm{a}$ and $1 \mathrm{~b}$ reveals that the thermal stability of the enzyme is not affected by the addition of sodium sulfate. However, supplementation of $25 \mathrm{mM}$ sodium bicarbonate results in an increase of approximately $5{ }^{\circ} \mathrm{C}$ in the melting temperature. a)

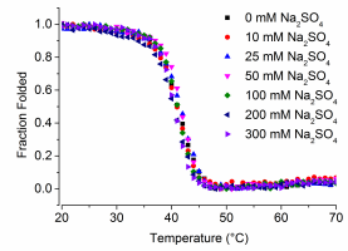

b)

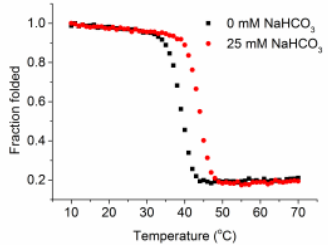

Figure 1. Thermal induced unfolding of $8 \mathrm{nM}$ OXA$143(\mathrm{P} 227 \mathrm{~S})$ varying the concentration of a) sulfate and b) bicarbonate.

\section{Conclusions}

The kinetic results indicate an increase in catalytic activity only in the assay with increasing bicarbonate concentration. Furthermore, the hydrodynamic parameters indicated that the enzyme is monomeric and has a globular or spherical tertiary structure under the studied conditions

\section{Acknowledgement}

FAEPEX (519.292, 3095/1 e 2391/18); CAPES; FAPESP; IQ-UNICAMP.

${ }^{1}$ Evans, B. A., Amyes, S. G., OXA $\beta$-lactamases. Clin. Microbiol. Rev., 2014,27, 241-263.

2Pinheiro, G.M.S., Ramos, C.H.I.., OXA-143, Initial characterization of newly identified mitochondrial and chloroplast small HSPs from sugarcane shows that these chaperones have different oligomerization states and substrate specificities. Plant Physiol. Biochem., 2018. 129, 285-294. 